\title{
SAÚDE MENTAL E ATENÇÃO BÁSICA:TERRITÓRIO, VIOLÊNCIA E O DESAFIO DAS ABORDAGENS PSICOSSOCIAIS
}

\author{
MENTAL HEALTH AND PRIMARY HEALTH CARE:TERRITORY, VIOLENCE AND THE PSYCHOSOCIAL \\ APPROACHES CHALLENGE
}

\section{SALUD MENTAL Y ATENCIÓN BÁSICA:TERRITORIO, VIOLENCIA Y EL DESAFÍO DE LOS ENFOQUES PSICOSOCIALES}

\author{
Nina Isabel Soalheiro dos Santos Prata ${ }^{1}$ \\ Daniel Groisman ${ }^{2}$ \\ Desiane Alves Martins ${ }^{3}$ \\ Elaine Teixeira Rabello ${ }^{4}$ \\ Flávio Sagnori Mota ${ }^{5}$
}

Resumo Este artigo teve como objetivo discutir os desafios para a implementação das ações de saúde mental na Estratégia Saúde da Família na perspectiva da desinstitucionalização e territorialização do cuidado. Descrevemos, na visão de gestores e equipes de saúde da família, o contexto territorial, formas de identificação das demandas e práticas de acolhimento e cuidado em saúde mental. Avaliamos ainda os desafios para a construção de abordagens psicossociais potentes e o cuidado em rede. O campo da pesquisa constituiu-se de dois territórios da cidade (Manguinhos e Complexo do Alemão) com características emblemáticas do contexto urbano do Rio de Janeiro no período estudado (2009 a 2013). Os pesquisadores trabalharam com estratégias de metodologias qualitativas e colaborativas que incluíram entrevistas com gestores, grupos focais com trabalhadores e visitas sistemáticas ao campo. Os dados coletados apontaram tendências específicas como expansão acelerada da Estratégia Saúde da Família com impactos no processo de trabalho; discurso dos gestores com evidência de abertura para inclusão da saúde mental na Estratégia; narrativas dos trabalhadores explicitando sensação de despreparo e baixa percepção do potencial terapêutico da atenção básica; violência nos territórios causando tensões e ambivalências em relação aos poderes locais; associação direta entre saúde mental e cotidiano violento.

Palavras-chave saúde mental; atenção básica; abordagens psicossociais; território.

\author{
Marco Aurélio Jorge ${ }^{6}$ \\ Mariana Lima Nogueira ${ }^{7}$ \\ Renata Ruiz Calicchio ${ }^{8}$ \\ Renata Veloso Vasconcelos ${ }^{9}$
}

Abstract This article aimed to discuss the challenges to the implementation of mental health actions in the Family Health Strategy in the context of deinstitutionalization and of the territorialization of care. We described, in the view of the family health managers and teams, the territorial context, forms of identification of the demands, and the mental health support and care practices. We also evaluated the challenges to building powerful psychosocial approaches and network care. The research field consisted of two territories in the municipality of Rio de Janeiro (Manguinhos and Complexo do Alemão), Brazil, with iconic features of the city's urban context, in the period studied (2009-2013). The researchers worked with qualitative and collaborative methodology strategies that included interviews with managers, focus groups with workers, and systematic visits to the field. The data collected showed specific trends, such as the accelerated expansion of the Family Health Strategy with impact on the work process; the discourse of the managers with evidence of opening for the inclusion of mental health in the Strategy; workers' narratives explaining their sense of unpreparedness and a low perception of the therapeutic potential of primary care; violence in the territories causing tension and ambivalence in relation to local authorities; direct association between mental health and violence in daily life.

Keywords mental health; primary care; psychosocial approaches; territory. 


\section{Introdução}

Este artigo apresenta os resultados de uma pesquisa 10 que abordou a relação entre território e adoecimento em saúde mental no contexto da Estratégia Saúde da Família (ESF) do município do Rio de Janeiro no período de 2009 a 2013, caracterizado por uma expansão acelerada de cobertura e implantação de um novo modelo de gestão. Os territórios estudados - Manguinhos e Complexo do Alemão - estão situados na Área Programática (AP) 3.1, uma das dez áreas definidas para regionalização da saúde na cidade. Trata-se de territórios com características específicas, mas que podem ser considerados emblemáticos de um contexto de grandes transformações urbanas no Rio de Janeiro, incluindo obras estruturais motivadas pelos grandes eventos na cidade, ocupação militar de áreas estratégicas e processos de intervenção do Estado para pacificação.

A pesquisa teve como foco as práticas de atenção em um cenário após 35 anos de Reforma Psiquiátrica Brasileira, 25 anos do Sistema Único de Saúde (SUS) e de uma escolha nacional por um modelo de saúde que prioriza a atenção básica e, em especial, a ESF. Partimos dos desafios comuns entre as políticas de atenção básica e saúde mental - ambas resultantes de movimentos de reforma nascidos ao longo do processo de redemocratização no país - para discutir a necessidade de uma articulação potente entre elas e sua convergência de princípios: a reorientação das práticas, a territorialização do cuidado e a importância do trabalho de equipe (Amarante, 2007; Jucá, Nunes e Barreto, 2009; Dalla Vecchia e Martins, 2009; Yasui, 2010; Penido, 2013). ${ }^{11}$

O objetivo geral do nosso estudo foi analisar as diretrizes da política, os impasses e os desafios para a inclusão e a implementação de ações de saúde mental e atenção psicossocial no contexto da ESF. Especificamente, visamos mapear e descrever demandas de cuidado em saúde mental nos territórios da Estratégia definidos como campo da pesquisa; identificar práticas territoriais de cuidado que poderiam ser potencializadas como abordagens psicossociais; e refletir sobre formas de construção de ações e redes em saúde mental.

\section{Atenção básica, saúde mental e o contexto do campo da pesquisa}

A cidade do Rio de Janeiro tinha em 2010 cerca de seis milhões de habitantes (Brasil, 2010) distribuídos em 160 bairros agrupados em dez áreas programáticas ou de planejamento (APs), divisão territorial dentro da qual encontramos desigualdades e situações paradoxais em relação aos indicadores sociais e de saúde (Brasil, 2015; Pinto, 2011). Quanto à organização 
dos serviços, a atenção à saúde no município se estrutura em três níveis com diferentes tipos de unidades: atenção primária (centro municipal de saúde/CMS e clínica da família/CF); atenção secundária (policlínicas, centro de atenção psicossocial/Caps, unidades de pronto atendimento/Upas e centros de reabilitação); e atenção terciária (maternidades, hospitais e institutos) (Rio de Janeiro, 2011; Organização Pan-Americana da Saúde, 2013).

A pesquisa teve como foco a atenção primária, mais especificamente nas unidades de saúde da família, que no Rio de Janeiro são denominadas 'clínicas da família' e identificadas como inovadoras e estratégicas para o atual modelo de gestão. Com a opção clara pela ESF como modelo organizador da atenção, a Secretaria de Saúde do município investiu fortemente na criação dessas clínicas, que se tornaram uma espécie de 'carro-chefe' da saúde no Rio de Janeiro.

A proposta, tal como apresentada pela gestão, seria qualificar a atenção, oferecendo infraestrutura sofisticada (ambiência, conforto, beleza e sustentabilidade), adequadamente equipada e com ofertas de procedimentos (exames laboratoriais, raios $\mathrm{X}$, ecografia, entre outros), além de inovações tecnológicas (Observatório de Tecnologia de Informação e Comunicação em Sistemas e Serviços de Saúde, prontuário eletrônico, acesso à internet e rede $w i$-fi), dentre outras características (Rio de Janeiro, 2011).

As sedes das clínicas da família estão localizadas em pontos estratégicos dos territórios sob sua responsabilidade, apresentando arquitetura e estética bem cuidadas. Em cada uma delas se instalam de cinco a mais equipes de saúde da família, compostas por médicos de família, enfermeiros, técnicos de enfermagem, cirurgiões-dentistas, técnicos de saúde bucal, todas com agentes comunitários de saúde (ACSs) e agentes de vigilância em saúde. Essas equipes são responsáveis pela atenção integral à saúde da população daquela área, assim como pelo cumprimento de metas e indicadores de resultados pactuados em contratos de parceria de gestão entre instituições da sociedade civil, denominadas de organizações sociais (OSs), e a Secretaria de Saúde/Secretaria Municipal de Defesa Civil do Rio de Janeiro (SMSDC-RJ), atual Secretaria Municipal de Saúde do Rio de Janeiro (SMS-RJ) (Organização Pan-Americana da Saúde, 2013).

Até 2009, ano de início do nosso estudo, o cenário da atenção básica no município apresentava uma estrutura bastante incipiente, com cobertura da ESF em torno de 7\% (Organização Pan-Americana da Saúde, 2013). Nessa época iniciou-se um processo de mudança na gestão da saúde, com ênfase na ESF e consequente estímulo financeiro, o que resultou numa expansão acelerada, com implantação de 828 equipes, atingindo uma cobertura de 47,9\% em março de 2015 (Brasil, 2015), ao se considerarem apenas equipes de saúde completas. Para viabilizar essa expansão de cobertura, vêm sendo implementadas no campo da gestão do trabalho algumas estratégias para 
atrair e fixar profissionais qualificados, incluindo alteração de salários e formas de contratação, operacionalizadas por meio das OSs. Outra estratégia importante está voltada para o campo da qualificação profissional em parceria com instituições de ensino, como a Universidade Federal do Rio de Janeiro (UFRJ), a Universidade do Estado do Rio de Janeiro (Uerj) e a Fundação Oswaldo Cruz (Pinto, 2011; Magnago e Pierantoni, 2015; Organização Pan-Americana da Saúde, 2013).

Outro passo foi dado a partir de 2010, com o início da implantação dos núcleos de atenção à saúde da família (Nasfs), que têm o objetivo de apoiar e dar resolutividade às ações de saúde na atenção básica, além de promover sua integração à rede de serviços. Uma rede de Nasfs foi progressivamente implementada, chegando a 47 equipes em 2015. Para responder à necessidade de estabelecer interlocuções entre ESF e Nasf, foram promovidas diversas oficinas de integração para organização do trabalho das equipes, de acordo com as especificidades de cada área (Brasil, 2015).

Embora o planejamento estratégico do município apresentasse a meta de atingir 100\% de cobertura da ESF até 2016 (Rio de Janeiro, 2013), o modelo de atenção à saúde municipal ainda não está consolidado e apresenta problemas com relação ao acesso, à coordenação e longitudinalidade do cuidado, assim como alta rotatividade de profissionais, especialmente de médicos (Magnago e Pierantoni, 2015; Organização Pan-Americana da Saúde, 2013).

Aliado a esse panorama, temos um contexto de profundas transformações urbanas relacionadas aos grandes eventos realizados na cidade (Copa do Mundo de 2014 e Olimpíadas 2016), ao protagonismo da população do Rio de Janeiro nas manifestações de junho de 2013 e aos processos de intervenção do Estado para pacificação, em especial as unidades de polícia pacificadora (UPPs). Um ambiente que apresenta tensões entre grupos políticos e gestão municipal, estadual e federal, trazendo impasses na percepção e execução de projetos para a cidade.

Em perspectiva histórica, a política nacional de saúde mental, construída no contexto do movimento social pela Reforma Psiquiátrica Brasileira, foi implementada contemporaneamente à política de saúde da família. São muitos os estudos que apontam as suas convergências, a necessidade de uma articulação potente entre elas e o papel estratégico para o acolhimento e cuidado (Figueiredo e Campos, 2009; Nunes, Jucá e Valentim, 2007; Tófoli e Fortes, 2007; Penido, 2013).

Ressaltamos, portanto, o caráter estratégico da relação entre saúde mental e saúde da família, considerando que a atenção básica tornou-se a porta de entrada preferencial de todo o sistema de saúde - inclusive das demandas de saúde mental -, além de coordenadora das ações de cuidado. Na mesma medida, no projeto contemporâneo da saúde mental para o SUS, a relação com o território passou a representar importante indicador de qualidade da 
rede e do tipo de transformação na lógica do cuidado que efetivamente se consegue operar (Quintas e Amarante, 2008).

Nessa perspectiva, as noções de 'território' e 'contexto' fundamentam uma compreensão da doença como manifestação do indivíduo, mas indissociável da situação de saúde do lugar, entendendo que os lugares e seus diversos contextos sociais são resultado de situações históricas, ambientais e sociais que promovem condições particulares para a produção de processos de adoecimento. Os territórios têm vida, expressa pela história de pessoas e lugares, pela cultura, pelos movimentos sociais e ações políticas, sendo a expressão de interesses muitas vezes conflitantes (Monken e Barcellos, 2007; Fernandes e Costa, 2009; Monken, Barcellos e Porto, 2011).

Nos debates do campo, a perspectiva da atenção psicossocial assume progressiva importância, compreendida como o conjunto de um novo modo de organização do cuidado, marcado pela diversidade conceitual e temática - que abrange o sofrimento psíquico como um processo complexo, o qual necessita de diferentes olhares e práticas interdisciplinares (Venâncio, Leal e Delgado, 1997; Amarante, 2007; Costa-Rosa e Yasui, 2000; Vasconcelos, 2008).

A concepção de saúde mental e a de atenção psicossocial, tais como entendidas neste estudo, estão sempre referidas à perspectiva da (des)institucionalização, conceito fundamental para o campo da reforma brasileira. Em Amarante (1996), encontramos indicações para pensar que o processo de desinstitucionalização implica uma ideia de a posteriori, de um futuro a ser construído, por meio da invenção de novas realidades e instituições. A desinstitucionalização, dessa forma, é um processo que não prescinde das instituições, mas as reinventa permanentemente, trabalhando contra estratégias seculares da institucionalização e sua cultura de exclusão.

\section{Metodologia e desenvolvimento da pesquisa}

A perspectiva metodológica utilizada neste estudo pode ser caracterizada como qualitativa e colaborativa, visto que priorizou a narrativa dos partícipes da pesquisa não apenas como atores envolvidos na realidade da qual falam, mas também como sujeitos singulares cujas narrativas foram incorporadas na análise dos dados e discussão de suas implicações para a constituição de redes de cuidado em saúde e saúde mental nos territórios estudados. Tal proposta pressupôs a escolha por estratégias participativas que incorporassem novos segmentos na produção de conhecimentos sistemáticos sobre as políticas e serviços (Furtado e Campos, 2008; Campos e Furtado, 2008).

O campo da pesquisa foi definido por cinco unidades de saúde da família localizadas no território da AP 3.1, tendo como critério para sua elegibilidade 
a vinculação dessas equipes ao Programa de Residência Multiprofissional em Saúde da Família da Escola Nacional de Saúde Pública Sergio Arouca, da Fundação Oswaldo Cruz. Foram incluídas duas unidades em Manguinhos e três unidades do Complexo do Alemão. O projeto desta pesquisa foi submetido aos comitês de ética em pesquisa da Secretaria Municipal de Saúde do Rio de Janeiro e da Escola Politécnica de Saúde Joaquim Venâncio, da Fundação Oswaldo Cruz, e devidamente aprovado, de acordo com o protocolo n. 001 2.0.408.314-11.

O estudo foi desenvolvido em duas etapas sucessivas e com metodologias complementares. Na primeira fase foram realizadas entrevistas semiestruturadas com gestores do nível central, da área programática e das cinco unidades de saúde da família, que constituíram o campo da pesquisa. Nelas foram identificados os princípios gerais da política, os impasses e desafios para sua implementação, assim como as articulações entre a ESF, a política e as ações de saúde mental no contexto da cidade do Rio de Janeiro e dos territórios estudados. Na sequência, foram realizadas entrevistas em grupo com as equipes de trabalhadores das unidades de saúde da família, que constituíram o campo da pesquisa para investigação das demandas, formas de acolhimento e cuidado em saúde mental.

Concomitantemente à realização das entrevistas, os pesquisadores acompanharam diversas ações de cuidado em rede desenvolvidas, com visitas regulares ao território, de acordo com as necessidades da pesquisa e sempre acompanhadas pelos ACSs.

Nossa escolha foi por um dispositivo de pesquisa que pudesse favorecer a participação e a colaboração direta dos profissionais no processo. Assim, tivemos como metas, além de coletar dados objetivos para a pesquisa, promover trocas entre o ambiente acadêmico e o campo da atenção, colaborar para o exercício de reflexão da equipe sobre seu processo de trabalho e contribuir para a desconstrução da concepção corrente de pesquisadores como avaliadores e neutros.

\section{Os territórios estudados: Manguinhos e Complexo do Alemão}

O território de Manguinhos é formado por 13 comunidades, com população estimada em cerca de 36.000 habitantes. Foi assim denominado por se tratar de uma região originalmente constituída por manguezal. A região iniciou seu processo de urbanização com a chegada da ferrovia em 1886, e o desenvolvimento local veio com a construção de novas vias que estimularam a instalação de instituições e empresas, como o Instituto Soroterápico, em 1900 (hoje Fundação Oswaldo Cruz), a Empresa Brasileira de Correios e Telégrafos (ECT), a Embratel, a Souza Cruz e a Refinaria de Manguinhos, entre outras. 
Até os anos 1940, a região se caracterizava como um bairro industrial, mas a partir dos anos 1950 foi alvo de grande fluxo migratório de outras áreas que sofreram políticas oficiais de remoção. Foram construídos grandes conjuntos habitacionais, ao mesmo tempo que se verificou um processo crescente de invasões e crescimento da violência urbana (Fernandes e Costa, 2009). Durante o período de realização da pesquisa, a região recebeu grande investimento do Programa de Aceleração do Crescimento (PAC) do governo federal, conjunto de políticas públicas para o crescimento econômico, criação de infraestruturas urbanas e de saneamento em todo o país. O PAC-Manguinhos foi lançado em 2008, e suas obras têm gerado fluxos migratórios e impactos importantes na vida das comunidades, como constatamos na análise das entrevistas.

Diversas comunidades constituem Manguinhos, com características geralmente definidas pelas origens da ocupação, incluindo áreas com perfis de adoecimento relacionados à extrema pobreza e áreas com melhores condições de urbanização. Essa diferenciação interna se reflete no perfil epidemiológico: o segundo grupo é caracterizado pela incidência de diabetes, hipertensão e doenças crônicas em geral; e o primeiro, por problemas ligados à falta de saneamento básico (escabiose, verminoses, impetigo), além do abuso de drogas, alcoolismo, gravidez precoce e doenças sexualmente transmissíveis (DSTs).

Uma das marcas do território sempre foi a presença do tráfico armado e ofensivo, a existência de 'cracolândias' e a ocupação dos espaços públicos de convivência - ruas, praças e alguns imóveis - pelo tráfico/uso de drogas. Nas áreas mais pobres, a urbanização é precária, constituída por ruelas e becos com moradias construídas em meio a escombros e lixo, um ambiente que causou grande impacto nos profissionais de saúde e nos pesquisadores nestes, especialmente, pelas demonstrações claras de hostilidade à sua presença, inclusive com a exibição de armas e demarcação de áreas de poder.

A ocupação para instalação de uma UPP aconteceu em janeiro de 2013. Nas idas ao campo, constatamos uma opressão generalizada, com relatos contundentes sobre os efeitos da violência no processo de trabalho dos profissionais de saúde e na produção de demandas de cuidado em saúde mental ao longo do processo de pacificação.

Em outra das subáreas estudadas encontramos uma realidade diferente, uma das raras regiões em que não havia tráfico. Havia saneamento e coleta regular de lixo, associação de moradores atuante, creche particular, escolas municipais, quadras, correios e igrejas, e a equipe se orgulhou da taxa zerada, na época, de casos de tuberculose. Uma situação que se apresentou como uma ilha de urbanização num entorno de miséria, em que pudemos observar marcos de separação defendidos pela população local, que demonstrava claramente não querer ser identificada com o conjunto da comunidade. 
Dessa forma, Manguinhos pode ser pensado como um conjunto de territórios múltiplos, com diversas origens e histórias de ocupação, uma população que convive com profundas transformações urbanas motivando fluxos migratórios e impactos na qualidade de vida e saúde, além de uma presença imperativa da violência. A cobertura da ESF é total (100\%), mas o relato das equipes evidenciou uma rede intersetorial precária.

No segundo território estudado, o Complexo do Alemão, os primeiros registros de ocupação datam do final da década de 1920, mas foi só nos anos 1960 que houve um crescimento populacional expressivo, também incentivado pelas indústrias na região. Nos anos 1990 se iniciou um processo de desindustrialização, acompanhado pela intensificação de ocupações irregulares, tráfico organizado e violência. A região é composta de um extenso território formado por 14 (15) comunidades, cujo índice de desenvolvimento humano (IDH) está entre os menores da cidade do Rio de Janeiro, segundo dados do último censo (Brasil, 2010). Também recebeu investimento do PAC embora não na mesma proporção de Manguinhos -, voltado para abertura e alargamento de vias dentro das favelas e no seu acesso, pavimentação de becos, construção de centros sociais, além da construção do conhecido teleférico do Alemão. As unidades de saúde têm localização periférica em relação à comunidade, distância que impõe desafios, dificultando as ações territoriais de saúde.

Historicamente é também um território ocupado por grupos armados, o que fez com que o trabalho de pesquisa fosse muito marcado por esse contexto - incluindo os momentos dramáticos da ocupação da região pelas forças de segurança em novembro de 2010, amplamente divulgada pela imprensa. Na época, cenas de confronto foram transmitidas ao vivo em rede nacional, causando as mais diversas reações e grande comoção popular. A intervenção possibilitou a instalação da primeira UPP local em abril de 2012. As ações para pacificação continuam se dando em meio a conflitos e mortes. Ao longo dos três anos de trabalho, a pesquisa apresentou retratos desses momentos, das diversas fases do processo de pacificação e dos diferentes tipos de tensões.

Conforme o período, os relatos dos sujeitos da pesquisa remeteram à presença de um tráfico ostensivo ou à violência da ocupação (ora por eles denominada de 'invasão', ora de 'ocupação'), quando houve a denúncia de atos abusivos não divulgados pela imprensa, ou ainda à queixa de serem vítimas de uma tensão permanente na convivência entre grupos armados. Foram muitos relatos emocionados e traumáticos, muitas vezes cortados pela emoção das lembranças, trazendo histórias de processos de adoecimento de profissionais e pacientes desencadeados pela violência no cotidiano.

Também foram descritos territórios e perfis de adoecimento diversos, desde aqueles cujos maiores problemas eram ligados ao lixo, escombros e 
infestação de roedores, até algumas poucas áreas com perfil de saúde associado às populações economicamente mais favorecidas, com doenças crônicas em geral, especialmente diabetes e hipertensão em idosos.

\section{A perspectiva dos gestores: abertura para a inclusão da saúde mental}

A análise das entrevistas com os gestores (um total de sete entrevistas individuais) apontou alguns desafios importantes para uma articulação potente entre atenção básica e saúde mental, todos enfatizando a complexidade que envolve gerir um sistema de saúde numa cidade como o Rio de Janeiro. Para os entrevistados, a violência urbana representava um desafio permanente, afetando as ações de gestão, o trabalho dos profissionais e os usuários do sistema de saúde.

O caso singular da violência na cidade do Rio de Janeiro tem sido analisado por diversos autores. Recorremos a alguns deles para discutir as questões relevantes e pertinentes aos territórios estudados, todos marcados pelos efeitos comuns da violência, suas formas de organização e seus subprodutos, que, como abordamos em outro momento (Soalheiro, 2012), impactam fortemente a rotina do trabalho em saúde mental.

Os gestores que atuavam na área ressaltaram uma mudança em relação à tradição de respeito e preservação das unidades de saúde, havendo agora uma maior vulnerabilidade dos profissionais, o que gerava medos e resistências por parte destes. O que acabou por afastá-los das idas à comunidade e visitas domiciliares, estas delegadas exclusivamente aos agentes comunitários. Os gestores de unidades comentaram também esse aspecto, apontando que os ACSs, por serem trabalhadores e também moradores, se submetiam a uma sobrecarga em relação às constantes pressões de um cotidiano violento. Por conhecerem as atividades ilegais no território e as pessoas envolvidas, apresentavam frequentemente uma visão ambivalente em relação à 'pacificação' e aos diferentes poderes locais. Ressentiam-se dos processos de ocupação, identificando-os como algo ameaçador e desconhecido, sem vislumbrar a perspectiva de uma vida sem violência.

Serafini (2013) cita alguns aspectos que foram implementados e definem a forma de gestão da cidade, dentre os quais ressaltamos o alto número de remoções; a política de internação compulsória dos moradores de rua, particularmente em nome do combate às drogas; o chamado 'choque de ordem'; a construção de barreiras físicas que separam favelas e tráfego nas vias expressas, por exemplo. Para esse autor, trata-se de um conjunto de ações que objetivariam conter a expansão e a visibilidade das favelas, além de aumentar o controle social sobre as populações pobres por meio do encarceramento, vigilância ou confinamento. 
Uma política pública para a qual se esperava um caráter de policiamento comunitário - modelo que comportaria uma perspectiva de gestão democrática da segurança pública, com participação da comunidade na definição das prioridades, estratégias e controle do policiamento - e que se torna cada vez mais distante de um ideal de pacificação e gestão de situações de conflito (Serafini, 2013). O que foi discutido também pelo estudo de Fleury (2012), realizado na primeira favela ocupada no Rio de Janeiro, no qual a autora demonstra as contradições entre a implantação de uma ordem policial coercitiva, a expansão dos direitos de cidadania dos moradores e sua integração à cidade.

Outro aspecto importante apontado pelos gestores foi a relação das transformações urbanas com sobrecarga de trabalho na saúde, o que causava dificuldades para a sistematização das ações e o acompanhamento longitudinal dos casos. No entanto, eles ressaltaram a importância das remoções de populações vulneráveis com o apoio do Estado por meio do aluguel social e da construção de áreas de moradias planejadas. Comentaram sobre as relações entre território e condições de vida como determinantes dos processos de adoecimento, mas sem problematizar a localização das unidades muitas vezes periféricas às comunidades. A polêmica da determinação de internação compulsória para os usuários de crack - questão em evidência na época - foi citada, mas admitiam-se divergências internas sobre o tema. Era consensual apenas a defesa de soluções sociais não centradas no modelo hospitalar.

As clínicas da família foram apontadas como inovadoras, na medida em que tinham uma infraestrutura moderna que possibilitava melhora na qualidade do atendimento, e não se reconheceu nelas o risco de reprodução do modelo médico-centrado. Os entrevistados referiram-se à rotatividade de gestores e outros profissionais como um desafio, mas sempre ressaltando a dimensão nacional desse problema e o fato de estarem conseguindo manter as equipes completas e ainda com aumento de cobertura. Defenderam a parceria com as organizações sociais como um modelo de gestão que tem possibilitado cumprir a meta de expansão, caracterizando-o como novo e radicalmente não hospitalocêntrico.

Os gestores argumentaram que o processo acelerado de aumento da cobertura dificultava o matriciamento, por demandar grande esforço de capacitação dos profissionais da ESF para o acolhimento das demandas de saúde mental. Mas demonstraram extrema abertura e sensibilidade para as abordagens ao sofrimento mental e sua inclusão na lógica de trabalho das equipes de saúde da família, com falas enfáticas em defesa da responsabilização pelo acolhimento e tratamento das demandas.

Os gestores mais diretamente envolvidos com a saúde mental explicitaram receios quanto à possibilidade de que o investimento na política e no modelo da atenção básica como gestora do cuidado trouxesse riscos de desin- 
vestimento no avanço da política de saúde mental no município. Apontaram dificuldades internas na medida em que reconheciam um movimento de resistência dos profissionais, em especial dos Caps, em legitimar a atenção básica como a porta de entrada do sistema, associando tal aspecto ao receio de perda do protagonismo na coordenação do cuidado na rede de saúde mental.

Essa questão também esteve presente na fala dos gestores mais diretamente ligados à ESF, que ressaltaram a importância de se priorizar a longitunalidade da atenção para a assistência, não apenas para a saúde mental, mas também para a construção de um vínculo em que cada pessoa saiba quem é o responsável pelo cuidado. Alguns gestores falaram da sua compreensão do modelo de saúde da família com um componente assistencial forte, de consultas, de tratamento de doenças. E destacaram a visão desse modelo como um sistema restrito à prevenção e promoção da saúde como um erro a ser superado. Identificaram a saúde mental como uma área da saúde que se isolou em um nicho, criando um sistema próprio, sem levar em consideração que a pessoa com problemas mentais sofre com outras demandas. Defenderam como indicador de um bom sistema de saúde a assistência em saúde mental incluída na atenção primária, com unidades especializadas apenas para casos mais graves.

Foi apontada a necessidade de se repensar a atualidade de priorização dos processos de desinstitucionalização, ampliando o acesso e as ações. Os gestores ressaltaram sua visão da Reforma Psiquiátrica como inserida num contexto muito forte de desinstitucionalização - e que esta não seria mais quantitativa e qualitativamente o maior problema atual. Eles foram enfáticos quanto à perspectiva de que as crises mais básicas e longitudinais deveriam ser atendidas pelo médico de família, pela equipe de saúde da família, em uma dinâmica comunitária.

Destacou-se assim, nas várias entrevistas com gestores, a crítica explícita ou implícita à estrutura normativa da política nacional de saúde mental vigente, que não dialogaria bem com a atenção primária como ordenadora do cuidado. Apontou-se a necessidade de revisão da função dos Caps como organizadores da rede, atribuindo a esta o caráter de um recorte histórico que tenderia a ser superado - ressaltando-se a necessidade de que o centro de atenção psicossocial retomasse seu lugar de dispositivo especializado para atenção integral intensiva e semi-intensiva. Mencionou-se como desafio a criação de uma rede de sustentação e agenciamentos locais para projetos de geração de renda e inserção no trabalho, de acesso à moradia digna e também de redução do estigma.

A violência sexual contra crianças foi citada em especial também como desafio, assim como a falta de recursos para apoiar pessoas envolvidas em acidentes, eventos com perdas abruptas e situações graves de violência. Apontaram-se ainda os suicídios como grave problema, destacando-se a 
carência de políticas públicas para acompanhamento das tentativas de suicídio como eventos importantes e a necessidade de mobilização de recursos para criar redes de vigilância, suporte e atenção às pessoas e famílias envolvidas.

Criticou-se a formulação atual do conceito de matriciamento, caracterizando-o como insuficiente para definir a dimensão do diálogo necessário e permanente das equipes de saúde da família com os profissionais especializados em saúde mental para o acompanhamento de casos. Nesse sentido, apontou-se como desafio a construção de competências para uma escuta mais qualificada para conduzir intervenções, reduzir atitudes prescritivas, compreender o sujeito vulnerável em sua forma própria de organizar a vida e seu universo simbólico. Desse modo, os gestores entrevistados ressaltaram, sobretudo, a importância de o profissional da atenção básica dialogar com a equipe de saúde mental e vice-versa - e de que os dois lados assumissem um papel de agenciamento da rede local, tendo como perspectiva a melhoria das condições de vida e saúde para os seus usuários que apresentassem sofrimento e transtornos mentais.

\section{A perspectiva dos trabalhadores: potência e impotência}

As equipes que atuam em Manguinhos ressaltaram os impactos das obras do PAC no seu trabalho e nas comunidades, entre eles a dificuldade de circulação nos territórios e os constantes fluxos migratórios das populações, o que provoca transformações na delimitação das áreas de abrangência e desafios para o acompanhamento longitudinal. Enfatizaram o sofrimento das famílias nos processos de remoções, agravado pela insegurança quanto à garantia de nova moradia, pela violência e pelas condições de vida empobrecidas diante da escassez de espaços públicos de lazer e convivênciaaspecto corroborado e tratado de forma aprofundada por Pivetta, Zancan e Guimarães (2012) em Manguinhos: um relato fotográfico, 2008-2010.

Esses autores salientam que buscaram retratar aquilo que muda e o que permanece num contexto de grandes transformações no complexo de favelas de Manguinhos, antes chamado de 'Faixa de Gaza'. São apresentados aspectos dessas transformações que se relacionam com os determinantes sociais da saúde, por isso afetam as pessoas, os lugares e a saúde do território: moradia, transporte, saneamento, lazer, educação e cultura, empregabilidade. Encontramos no olhar dos autores algo muito presente nas narrativas dos sujeitos da pesquisa: Manguinhos como um lugar que contém a diversidade e a heterogeneidade próprias dos espaços urbanos nas metrópoles, onde a provisoriedade e a precariedade são características importantes, mas que não excluem os sentimentos de pertencimento, enraizamento e orgulho dos que ali construíram suas vidas. 
O PAC e suas obras são identificados pelos autores como uma política pública fragmentada, que não articula as diferentes esferas de governo; porém, mesmo sem a perspectiva da intersetorialidade, seu conjunto de ações busca olhar para o território de forma mais ampla, com uma visão menos segmentada. Ainda que com vários limites, o PAC buscaria enfrentar os determinantes sociais da saúde investindo na construção de equipamentos sociais nas áreas de educação, cultura, esporte e lazer, entre outras, contribuindo para a promoção da saúde e a justiça social e ambiental. Pivetta, Zancan e Guimarães (2012) apontam problemas como a distância entre o anunciado pelas autoridades e o realizado, a duvidosa qualidade das obras de habitação e de saneamento básico, o déficit habitacional e a desterritorialização de moradores.

Por meio de imagens e reflexões muito presentes também em nossa pesquisa, o trabalho oferece imagens da intervenção urbana em Manguinhos, constituindo-se como um relato/narrativa da história de reivindicações e conquistas dessa comunidade, das suas múltiplas instituições, territorialidades e sociabilidades. São dados que nos possibilitam entender o que representa morar em Manguinhos, nos aproximar do cotidiano daqueles que construíram ali suas vidas afetivas e sociais, com suas violências e carências de infraestrutura, suas possibilidades e impossibilidades resultantes de políticas públicas geradas numa sociedade desigual e injusta.

Nas idas ao território, constatamos também a relevância dos problemas relacionados ao uso prejudicial de álcool e outras drogas, incluindo a visão inusitada de 'uma espécie de tapete humano' que se formava, na época, no entorno do serviço de saúde local - dezenas de pessoas deitadas no chão, abrigando-se no único lugar em que se sentiam protegidas para descansar ou dormir. Além disso, Manguinhos sediava alguns locais que se constituíram como cenas de uso de drogas, as chamadas cracolândias - característica que fez com que a comunidade fosse apropriadamente nomeada como 'território-cracolândia' num dos relatos da pesquisa. Uma realidade que não é mais a mesma em razão das migrações dos usuários e cenas de uso após intervenções do Estado, que envolveram recolhimento e internações involuntárias amplamente polemizadas e cuja discussão ultrapassa os limites deste artigo.

As entrevistas evidenciaram - dado verificado nos dois territórios estudados - uma queixa generalizada dos trabalhadores sobre falta de tempo para discussão e sistematização dos casos em equipe, o que levou à constituição de espaços informais para a realização deste trabalho. Falou-se sobre um excesso de demandas da gestão por registros estatísticos e preenchimentos de fichas de atendimento, o que reduzia o tempo destinado aos grupos e projetos terapêuticos. Dentre os temas relativos ao processo de trabalho, destacaram-se o sofrimento da equipe com a convivência num cotidiano violento, o medo de adoecer, os casos de afastamento do trabalho e suas demandas em relação à criação de espaços de acolhimento e cuidado para os profissionais. 
Os entrevistados referiram-se ainda ao sentimento de impotência em relação ao ciclo vicioso de gravidez na adolescência, trazido pela constatação de que, apesar de todo o trabalho de prevenção realizado pelas equipes, o comportamento se repetia em sucessivas gerações de mães e filhas. Relatos associaram a gravidez precoce das adolescentes à baixa expectativa de vida dos jovens companheiros e pais dos seus filhos; também houve a associação entre gravidez proveniente de pais líderes do tráfico na comunidade e ascensão na escala social. Descreveu-se como situação dramática o acompanhamento de grande número de idosos em condições precárias de vida ou em situação de abandono, somando-se aos casos de depressão em cuidadores relacionados à sobrecarga e à falta de perspectivas para suas próprias vidas.

Quanto às demandas de saúde mental, foi evidenciada uma dificuldade inicial de identificá-las e caracterizá-las, muitas vezes associando-as a comportamentos repetidos e irracionais que causavam incômodos. Os entrevistados reconheceram a importância de se escutarem esses pacientes, mas relataram que eram poucos os que se sentiam disponíveis, o que tornou evidente a dificuldade de articulação de estratégias comuns. Apesar dessa constatação, identificaram casos em que conseguiram fazer algum acompanhamento, não obstante a visão de que a equipe não sabia lidar com essas demandas, assumindo o encaminhamento à psicologia como primeira e às vezes única alternativa. Também encontramos, no entanto, alguns relatos de tentativas de inserção dos pacientes nas atividades de promoção da saúde, academia cariocal4 e outras práticas grupais.

O relato das equipes do Complexo do Alemão, como já ressaltado, foi muito marcado pelo impacto da intervenção no território pelas forças de segurança do Estado, com aspectos ambivalentes quanto à caracterização como 'pacificação', 'invasão', 'ocupação'. Parte da equipe reconheceu mudança para melhor, e outra referiu-se às imagens divulgadas pela mídia com afirmações do tipo "tudo que apareceu na televisão não foi o que aconteceu" ou "só mostraram a parte boa" (sic).

Particularmente, os trabalhadores que moram na área relataram não se sentir seguros, afirmando até que o medo teria se tornado maior após a ocupação. Tinham medo de sair de casa, dificuldades para dormir. Relataram episódios de tensão e de como eram afetados pelos tiroteios iminentes; descreveram situações em que constatavam as relações entre violência e adoecimento; falaram de estresse; contaram casos envolvendo vizinhos e parentes em que havia o desencadeamento de sintomas psiquiátricos e surtos psicóticos. Também lembraram a dificuldade de retomar o trabalho após os dias iniciais da ocupação e descreveram casos de profissionais e pacientes que apresentavam sucessivos picos hipertensivos. 
Havia uma tensão evidente entre os profissionais, ocasionada por visões diferentes do processo, que provocava uma divisão entre os que apenas trabalhavam e os que viviam e trabalhavam no território, em especial alguns ACSs, não todos. As diferenças diziam respeito aos custos e benefícios da ocupação, ao grau de exposição às violências, às desigualdades entre eles, traduzidas na clássica oposição entre "morro"' e "asfalto"" (sic). Mesmo nos momentos em que afirmavam o fim do tráfico explícito, falavam da presença da violência relacionada a ele, agora de forma diferenciada: "A gente não escuta um tiro, mas vê cadáver esfaqueado, enforcado" (sic). E também de um pacto de silêncio que ainda persistiria entre todos.

As equipes referiram-se a queixas difusas e problemas emocionais por parte dos pacientes, identificando alguns como "poliqueixosos" (sic), avaliando que estes não teriam consciência da relação entre os sintomas e as vivências relacionadas ao cotidiano violento. Relataram sua percepção de uma importante demanda de pessoas pelo uso prejudicial de álcool e drogas, referindo-se a esses casos como "muito difíceis" e estigmatizados. Além disso, falaram de histórias de vida dramáticas, diante das quais se sentiam impotentes e com ímpeto de "chorar junto" (sic), por não saberem o que fazer, como lidar com a situação. Sentiam-se de certa forma culpados, em especial os agentes comunitários, por estarem atuando "sem ter uma capacitação" (sic) para isso. Observamos que alguns dos ACSs acabavam assumindo um papel de mensageiros da equipe, agentes de marcação de consulta, mediadores da falta.

Apesar disso, ouvimos muitas referências àquilo que aparece na fala de um profissional como uma "escuta sem método" (sic). Seriam espaços abertos para a conversa, a escuta da história de vida, o acolhimento do paciente que sofre. Referiam-se muito positivamente à prática do matriciamento feita por profissionais do centro de atenção psicossocial, as consultas e abordagens conjuntas, mas continuaram afirmando sua insegurança e seu sentimento de despreparo para lidar com os casos de saúde mental - aspecto importante e que necessitaria de um estudo específico e melhor compreensão por parte das equipes e dos especialistas em saúde mental.

No relato das equipes, constatamos a centralidade da violência como fator de produção de adoecimentos e vulnerabilidades, o que colocou em pauta o desafio que representa construir práticas em saúde mental na ESF que não produzam a medicalização da violência e sejam abordagens psicossociais potentes (Soalheiro e Mota, 2014). Para a saúde mental, esse diálogo com a saúde da família surge como essencial para a desinstitucionalização do próprio campo. O encontro entre as propostas da territorialização em saúde e dos processos de (des)institucionalização na saúde mental pode trazer novas perspectivas para ambas. 


\section{Considerações finais}

Costa-Rosa e Yasui (2000) e Vasconcelos (2008) definem as abordagens psicossociais como aquelas que articulam os fenômenos psicológicos com as outras dimensões da vida, sem reproduzir práticas terapêuticas propostas pelas abordagens clínicas convencionais, considerando o contexto histórico, os lugares e territórios. Para isso, os autores ressaltam a necessidade da construção de abordagens que incorporem os impasses do sofrimento subjetivo no cotidiano vivido em territórios complexos, que trabalhem a ampliação da autonomia e visem a um diálogo permanente com as equipes de saúde.

Como vimos, os resultados da nossa pesquisa apontaram relações entre os processos de adoecimento e as características dos territórios estudados. Como ressalta Fleury (2012), os processos de ocupação territorial de caráter militar e policial, característicos dos territórios estudados, são processos sociais que evidenciam tensões e contradições, produzindo aquilo que se define como uma militarização do campo social - característica que fica bastante evidente na análise dos dados da pesquisa.

As formas de adoecimento que advêm da exposição constante ao cotidiano violento de profissionais e pacientes nos territórios estudados reforçam a importância do reconhecimento pelos profissionais de saúde das relações entre elas e as vulnerabilidades de grupos e comunidades. Para o campo da saúde mental, esse diálogo com a saúde da família surge como essencial para a desinstitucionalização do próprio campo, mediante o reconhecimento de que os territórios promovem condições particulares para a produção de doenças, o que implica entender a cultura, as relações de poder e as forças políticas em conflito (Fleury, 2012).

Destacamos como ponto importante a localização periférica das unidades em relação às comunidades, o que faz com que as ações de saúde sejam pouco territorializadas. Com a distância entre comunidade e equipe, tende-se a fortalecer o lado 'clínica' em detrimento da perspectiva de 'estratégia' no contexto das ações da saúde da família - o que pudemos observar na tendência dos profissionais de trazerem para a unidade atividades desejáveis de serem realizadas no território, como rodas comunitárias e práticas grupais de promoção da saúde.

Com relação ao trabalho das equipes, identificamos pouca percepção do potencial das suas diversas abordagens e pouco uso do potencial dos espaços públicos e processos coletivos, incluindo as diversas práticas grupais. Dessa forma, a pesquisa apontou a necessidade de investimento na sensibilização e formação dos profissionais para gerar mais potência no trabalho desenvolvido, o que implicaria também trabalhar os modos de acolhimento e o estabelecimento de comunicações entre a atenção individualizada e as abordagens psicossociais. 
Constatamos uma rede intersetorial precária, matriciamento centrado nos Caps ou ausência de recursos na falta destes, falta de referência ambulatorial e Nasf, além de encaminhamentos individuais para psicologia e psiquiatria como alternativa fortemente presente no imaginário dos profissionais da atenção básica. A pesquisa apontou também a relevância das demandas relacionadas ao uso abusivo de álcool e outras drogas, em especial o crack, associado ao contexto da violência e da política da guerra às drogas.

Em perspectiva mais geral, a medicalização dos comportamentos e da vida, na contramão de uma lógica da saúde responsável e ética, é um risco para a interface saúde mental e atenção básica (Soalheiro e Mota, 2014). Novas abordagens do sofrimento psíquico e melhor acolhimento das necessidades daqueles que sofrem e demandam cuidados poderiam se dar mediante o reconhecimento da importância do lugar onde vivem, onde constroem suas identidades, buscam recursos para lidar com a dor e a solidão, onde tecem redes de relações e inclusão social.

Os dados apresentados neste artigo apontaram relações entre saúde mental e as condições de vida em territórios onde as equipes se deparam cotidianamente com importantes transformações advindas de intervenções urbanas, ações do Estado para pacificação e deslocamento das populações. Dessa forma, esperamos contribuir para o debate da inclusão das ações de saúde mental na ESF no Rio de Janeiro, em uma perspectiva da saúde mental na rede pública pensada dentro dos territórios e indissociável das condições de vida nos lugares.

\section{Colaboradores}

Todos os autores participaram do desenvolvimento da pesquisa em suas duas fases, realizando as atividades de campo, sistematização dos dados coletados, análise dos resultados e redação do artigo científico. A primeira autora foi responsável pela coordenação do trabalho em equipe. 
Resumen Este artículo tuvo como objetivo discutir los desafíos para la implementación de las acciones de salud mental en la Estrategia Salud de la Familia en la perspectiva de la desinstitucionalización y territorialización del cuidado. Describimos, en la visión de gestores y equipos de salud de la familia, el contexto territorial, formas de identificación de las demandas y prácticas de acogida y cuidado en salud mental. Evaluamos también los desafíos para la construcción de enfoques psicosociales potentes y el cuidado en red. El campo de la investigación estuvo constituido por dos territorios del municipio de Río de Janeiro (Manguinhos y Complexo do Alemão), Brasil, con características emblemáticas del contexto urbano de la ciudad, en el período estudiado (2009 a 2013). Los investigadores trabajaron con estrategias de metodologías cualitativas y colaborativas que incluyeron entrevistas con gestores, grupos focales con trabajadores y visitas sistemáticas al campo. Los datos recolectados señalaron tendencias específicas como expansión acelerada de la Estrategia Salud de la Familia con impactos en el proceso de trabajo; discurso de los gestores con evidencia de apertura para inclusión de la salud mental en la Estrategia; narrativas de los trabajadores explicitando sensación de falta de preparación y baja percepción del potencial terapéutico de la atención básica; violencia en los territorios causando tensiones y ambivalencias con relación a los poderes locales; asociación directa entre salud mental y violencia cotidiana.

Palabras clave salud mental; atención básica; enfoques psicosociales; territorio.

\section{Notas}

1 Fundação Oswaldo Cruz, Escola Politécnica de Saúde Joaquim Venâncio, Rio de Janeiro, RJ, Brasil.

$<$ ninasoalheiro@fiocruz.br>

Correspondência: Rua General Cristóvão Barcelos, 31, apto. 101, Laranjeiras, CEP 22245-110, Rio de Janeiro, RJ, Brasil.

2 Fundação Oswaldo Cruz, Escola Politécnica de Saúde Joaquim Venâncio, Rio de Janeiro, RJ, Brasil.

$<$ dgroisman@fiocruz.br>

3 Secretaria Municipal de Saúde de Duque de Caxias, Duque de Caxias, RJ, Brasil.

<desiane@yahoo.com.br>

4 Universidade do Estado do Rio de Janeiro, Instituto de Medicina Social, Rio de Janeiro, RJ, Brasil.

$<$ mcarelaine@hotmail.com>

5 Universidade do Estado do Rio de Janeiro, Rio de Janeiro, RJ, Brasil.

$<$ fsagnori@gmail.com>

6 Fundação Oswaldo Cruz, Escola Politécnica de Saúde Joaquim Venâncio, Rio de Janeiro, RJ, Brasil.

$<$ maurelio@fiocruz.br>

7 Fundação Oswaldo Cruz, Escola Politécnica de Saúde Joaquim Venâncio, Rio de Janeiro, RJ, Brasil.

$<$ mnogueira@fiocruz.br> 
8 Fundação Oswaldo Cruz, Escola Nacional de Saúde Pública Sergio Arouca, Rio de Janeiro, RJ, Brasil.

$<$ renata.ruizcalicchio@gmail.com>

${ }^{9}$ Fundação Oswaldo Cruz, doutoranda Escola Nacional de Saúde Pública Sergio Arouca, Rio de Janeiro, RJ, Brasil.

$<$ renatavv04@gmail.com>

10 A pesquisa foi realizada pelo Grupo de Pesquisa Desinstitucionalização, Políticas Públicas e Cuidado, da Escola Politécnica de Saúde Joaquim Venâncio, da Fundação Oswaldo Cruz, com financiamento da Fundação Carlos Chagas Filho de Amparo à Pesquisa do Estado do Rio de Janeiro (Faperj), por meio do Programa Pesquisa para o SUS: gestão compartilhada em saúde (PPSUS) (2009-2013), parceria Faperj/Conselho Nacional de Desenvolvimento Científico e Tecnológico (CNPq)/Secretaria Estadual de Saúde (SES).

11 Optamos por adotar a expressão 'atenção básica' para designar esse primeiro nível de atenção, acompanhando os documentos oficiais do setor saúde no Brasil (Brasil, 2006, 2011).

12 A chamada 'Academia carioca' é um programa de promoção da saúde, implantado pela SMS/RJ desde 2009, centrado na inserção da prática de atividade física regular nas unidades básicas de saúde e em ações comunitárias voltadas ao estímulo de um estilo de vida mais saudável e prevenção de doenças crônicas não transmissíveis.

\section{Referências}

AMARANTE, Paulo. O homem e a serpente: outras histórias para a loucura e a psiquiatria. Rio de Janeiro: Editora Fiocruz, 1996.

AMARANTE, Paulo. Saúde mental e atenção psicossocial. Rio de Janeiro: Editora Fiocruz, 2007.

BRASIL. Ministério da Saúde. Secretaria de Atenção Básica. Departamento de Atenção Básica. Política Nacional de Atenção Básica 2006. Brasília: Ministério da Saúde, 2006. (Série Pactos pela Saúde 2006, v. 4). Disponível em: <http://bvsms.saude.gov.br/bvs/ publicacoes/politica_nacional_atencao_ basica_2006.pdf>. Acesso em: 7 jan. 2014.

BRASIL. Ministério do Planejamento, Orçamento e Gestão. Instituto Brasileiro de Geografia e Estatística (IBGE). Contagem Populacional. XII Censo Demográfico, 2010. Disponível em: <http://censo2010.ibge.gov.br/sobre-censo. html >. Acesso em: 14 jul. 2015.

BRASIL. Ministério da Saúde. Departamento de Atenção Básica. Portaria n. 2.488, de 21 de outubro de 2011. Aprova a Política Nacional de Atenção Básica, estabelecendo a revisão de diretrizes e normas para a organização da atenção básica para a Estratégia Saúde da Família (ESF) e o Programa Agentes Comunitários de Saúde (Pacs). 2011. Diário Oficial da União, Brasília, DF, 21 out. 2011. Disponível em: <http://bvsms.saude.gov.br/ bvs/saudelegis/gm/2011/prt2488_21_10_ 2011.html>. Acesso em: 19 jan. 2014.

BRASIL. Ministério da Saúde. Sistemas de Atenção Básica e Departamento de Atenção Básica. Teto, credenciamento e implantação das estratégias de agentes comunitários de saúde, saúde da família e saúde bucal. 2015. 
Disponível em: <http://dab.saude.gov.br/ portaldab/historico_cobertura_sf.php $>$. Acesso em: 5 ago. 2015.

CAMPOS, Rosana T. O.; FURTADO, Juarez P. Narrativas: utilização na pesquisa qualitativa em saúde. Revista de Saúde Pública, São Paulo, v. 42, n. 6, p. 1.090-1.096, 2008.

COSTA-ROSA, Abílio; YASUI, Silvio O modo psicossocial: um paradigma das práticas substitutivas ao modo asilar. In: AMARANTE, Paulo (org.). Ensaios: subjetividade, saúde mental e sociedade. Rio de Janeiro: Editora Fiocruz, 2000. p. 141-168.

DALLA VECCHIA, Marcelo D.; MARTINS, Sueli T. F. Desinstitucionalização dos cuidados a pessoas com transtornos mentais na atenção básica: aportes para a implementação de ações. Interface: Comunicação, Saúde, Educação, Botucatu, v. 13, n. 28, p. 151-164, 2009.

FERNANDES, Tania M.; COSTA, Renato G. R. Histórias de pessoas e lugares: memórias das comunidades de Manguinhos. Rio de Janeiro: Editora Fiocruz, 2009.

FIGUEIREDO, Mariana D.; CAMPOS, Rosana. O. Saúde mental na atenção básica à saúde de Campinas, SP: uma rede ou um emaranhado? Ciência \& Saúde Coletiva, Rio de Janeiro, v. 14, n. 1, p. 129-138, fev. 2009.

FLEURY, Sonia. Militarização do social como estratégia de integração: o caso da UPP do Santa Marta. Sociologias, Porto Alegre, v. 14, n. 30, p. 194-222, ago. 2012.

FURTADO, Juarez P.; CAMPOS, Rosana O. Participação, produção de conhecimento e pesquisa avaliativa: a inserção de diferentes atores em uma investigação em saúde mental. Cadernos de Saúde Pública, Rio de Janeiro, v. 24, n. 11, p. 2.671-2.680, nov. 2008.

JUCÁ, Vládia J. S.; NUNES, Monica O.; BARRETO, Suely G. Programa de Saúde da Família e Saúde Mental: impasses e desafios na construção da rede. Ciência \& Saúde Coletiva, Rio de Janeiro, v. 14, n. 1, p. 173-182 , fev. 2009.
MAGNAGO, Carinne; PIERANTONI, Celia R. Dificuldades e estratégias de enfrentamento referentes à gestão do trabalho na Estratégia Saúde da Família, na perspectiva dos gestores locais: a experiência dos municípios do Rio de Janeiro (RJ) e Duque de Caxias (RJ). Saúde em Debate, Rio de Janeiro, v. 39, n. 104, p. 9-17, mar. 2015.

MONKEN, Mauricio; BARCELLOS, Christovam. O território na promoção e vigilância em saúde. In: FONSECA, Angélica F.; CORBO, Ana M. D. (orgs.). O território e o processo saúde-doença. Rio de Janeiro: EPSJV/Fiocruz, 2007. p. 51-86.

MONKEN, Mauricio; BARCELLOS, Christovam; PORTO, Marcelo F. S. Saúde, trabalho, ambiente e território: contribuições teóricas e propostas de operacionalização. In: MINAYO, Carlos G.; MACHADO, Jorge M. H.; PENA, Paulo G. L. (orgs.). Saúde do trabalhador na sociedade brasileira contemporânea. Rio de Janeiro: Editora Fiocruz, 2011. p. 161-180.

NUNES, Monica; JUCÁ, Vládia J.; VALENTIM, Carla P. B. Ações de saúde mental no Programa Saúde da Família: confluências e dissonâncias das práticas com os princípios das reformas psiquiátrica e sanitária. Cadernos de Saúde Pública, Rio de Janeiro, v. 23, n. 10, p. 2.375-2.384, 2007.

ORGANIZAÇÃO PAN-AMERICANA DA SAÚDE (OPAS). Organização Mundial da Saúde (OMS). Reforma da atenção primária à saúde na cidade do Rio de Janeiro: avaliação dos primeiros três anos de clínicas da família - pesquisa avaliativa sobre aspectos de implantação, estrutura, processo e resultados das clínicas da família na cidade do Rio de Janeiro. In: HARZHEIM, Erno (org.). Porto Alegre: Opas/OMS, 2013. Disponível em: <www. sbmfc.org.br/media/file/reforma_atencao. pdf $>$. Acesso em: 12 jul. 2015.

PENIDO, Claudia M. F. Apoio matricial em saúde mental no contexto da saúde coletiva. In: PAULON, Simone; NEVES, Rosana (orgs.). Saúde mental na atenção básica: a territorialização do cuidado. Porto Alegre: Sulina, 2013. p. 17-38. 
PINTO, Luiz P. Clínicas da família na cidade do Rio de Janeiro: a expansão da atenção primária em saúde em questão. Rio de Janeiro: Subsecretaria de Atenção Primária, Vigilância e Promoção da Saúde/Secretaria Municipal de Saúde do Rio de Janeiro, 2011. Disponível em: <http://congressoanterior. redeunida.org.br/resumos/RE1539-1.pdf $>$. Acesso em: 7 jun. 2011.

PIVETTA, Fatima; ZANCAN, Lenira; GUIMARÃES, Gleide. PAC Manguinhos: um relato fotográfico, 2008-2010. Rio de Janeiro: Ensp/Fiocruz, 2012. Disponível em: <www. conhecendomanguinhos.fiocruz.br/files/ rf-livrocompleto-impressao.pdf > . Acesso em: 24 out. 2016.

QUINTAS, Renata M.; AMARANTE, Paulo. Ação territorial do centro de atenção psicossocial em sua natureza substitutiva. Saúde em Debate, Rio de Janeiro, v. 32, n. 78/80, p. 99-107, dez.-jan. 2008.

RIO DE JANEIRO (Prefeitura). Secretaria Municipal de Saúde e Defesa Civil (SMSDC). Superintendência de Atenção Primária. Carteira de serviços: relação de serviços prestados na atenção primária à saúde. Guia de Referência Rápida. Rio de Janeiro: SMSDC, 2011. Disponível em: <www.rio.rj.gov.br/ dlstatic/10112/137240/DLFE-228987.pdf/1.0>. Acesso em: 8 jan. 2015.

RIO DE JANEIRO (Prefeitura). Pós 2016: o Rio mais integrado e competitivo. Plano Estratégico da Prefeitura do Rio de Janeiro 2013-2016. Rio de Janeiro: Prefeitura do Rio de Janeiro, 2013. Disponível em: <www. rio.rj.gov.br/dlstatic/10112/2753734/DLFE241955.pdf/Planejamentoestrategico2.0.1.3. 2.0.1.6..pdf $>$. Acesso em: 13 jan. 2014.
SERAFINI, Flávio. As unidades de polícia pacificadora e a segurança pública do estado do Rio de Janeiro entre a guerra e a paz. In: CONGRESO DE LA ASOCIACIÓN LATINOAMERICANA DE SOCIOLOGÍA, 29. Santiago, 2013. $<$ http://actacientifica.servicioit.cl/biblioteca/ gt/GT4/GT4_SerafiniF.pdf>. Acesso em: 10 dez. 2013.

SOALHEIRO, Nina I. Surtado ou assustado? Algumas considerações sobre violência e cuidado no contexto dos serviços territoriais de saúde mental e atenção psicossocial. In: ALARCON, Sergio; JORGE, Marco A. S. (orgs.). Rio de Janeiro: Editora Fiocruz, 2012. p. 283-297.

SOALHEIRO, Nina I.; MOTA, Flavio S. Medicalização da vida: doença, transtornos e saúde mental. Revista Polis e Psique, Porto Alegre, v. 4, n. 2, p. 65-85, 2014.

TÓFOLI, Luiz F.; FORTES, Sandra. O apoio matricial de saúde mental na atenção primária no município de Sobral, CE: o relato de uma experiência. Sanare, Sobral, v. 6, n. 2, p. 34-42, 2007.

VASCONCELOS, Eduardo M. Abordagens psicossociais. São Paulo: Hucitec, 2008.

VENÂNCIO, Ana T.; LEAL, Erotildes M.; DELGADO, Pedro G. O campo da atenção psicossocial. Rio de Janeiro: Te Corá Editora, 1997.

YASUI, Silvio. Rupturas e encontros: desafios da reforma psiquiátrica brasileira. Rio de Janeiro: Editora Fiocruz, 2010.

Recebido em 25/05/2015

Aprovado em 02/06/2016 